\title{
Jumps in High-Frequency Data on the Chinese Stock Market
}

\author{
Ying Li, Tengfei Jiang \\ Business School, Sun Yat-sen University, Guangzhou, China \\ Email: mnsliy@mail.sysu.edu.cn
}

How to cite this paper: Li, Y. and Jiang, T.F. (2017) Jumps in High-Frequency Data on the Chinese Stock Market. Journal of Mathematical Finance, 7, 467-490. https://doi.org/10.4236/jmf.2017.72025

Received: April 17, 2017

Accepted: May 28, 2017

Published: May 31, 2017

Copyright (C) 2017 by authors and Scientific Research Publishing Inc. This work is licensed under the Creative Commons Attribution International License (CC BY 4.0).

http://creativecommons.org/licenses/by/4.0/

\begin{abstract}
This study adopts two nonparametric methods, the activity signature function (ASF) and ratio analysis of cojumps, to test jumps in China's stock market. Jumps in the stock price, stock-index futures, and volatility of China Securities Index (CSI) 300 index are analyzed using data on the continuous main-contract price of the index. The findings are as follows. First, in the long run, the CSI 300 index process is a continuous process exhibiting jumps at all sampling intervals. In the short run, the index becomes a pure-jump process in times of recession while exhibiting the characteristics of a continuous or even semimartingale process in certain intervals. Second, the stock-index futures process is a continuous process with jumps at all sampling intervals and, in the short run, exhibits the characteristics of a pure-jump process every 6 months. Moreover, the volatility process generally exhibits the characteristics of pure-jump processes. Third, the CSI 300 index price process and the continuous main-contract price process of the CSI 300 stock-index futures are significantly and positively related, with jumps occurring with a time lag of less than 5 minutes; by contrast, the volatility and price processes of the index are nonsignificantly related.
\end{abstract}

\section{Keywords}

High-Frequency Data, Activity Signature Function, Cojump, Nonparametric Method

\section{Introduction}

Discontinuities in asset prices and fluctuations in price are referred to as "jumps". They are associated largely with information impacts or human factors. Theoretically, jumps in stock prices contradict the assumed continuity of asset prices. Capital asset pricing models may therefore vary depending on the form, strength, frequency, and extent of the jumps. Specifically, jump-diffusion models 
and pure jump models conflict with each other under stochastic processes; this increases difficulties in asset pricing, thereby affecting the management of risks, the selection of investment portfolios, and the pricing of derivatives. Moreover, stock-price jumps magnify risks. Risks in financial assets are typically divided into foreseeable risks (characterized by continuity) and unforeseeable risks (characterized by jumps); the predictability of the former exerts less of an influence on financial markets, whereas the unpredictability of the latter, coupled with limitations in the dissemination of information among the markets, can induce information asymmetry, and subsequently, power-law distributions, in the markets. Excessive fluctuations in risk-asset prices may cause delays in resource allocation. Thus, in research focusing on financial markets under stochastic processes, there has been increasing interest in investigations of jumps and fluctuations in asset prices, underlining the need to identify these jumps effectively.

High-frequency data are increasingly used in studies on financial market models under stochastic processes. These data are a component of the microstructures of financial markets, whose time-dependent asset prices suggest that the shorter a sampling interval is, the more information a sample contains. Their use has become increasingly prevalent among financial analysts because the continual advancements in computer technology over the past three decades has facilitated reducing the costs of the recording, storing, processing, and transmitting financial-market transaction data.

China's stock market is one of the most active emerging capital markets worldwide. It was established in the 1990s and exhibits unique characteristics compared with markets in Western countries with advanced economies that have been in effect for approximately two centuries. Information asymmetry causes fluctuations in stock markets, and thereby affects resource allocations in the markets. Compared with fluctuations in more mature stock markets, those in the Chinese stock market are larger and occur at a higher frequency, largely because of the immaturity of the market, which is currently in a phase of emergence and transition, exhibiting notable "policy market", temporal, and opportunistic characteristics. Moreover, the irrationality of the market structure renders market prices subject to volatility and frequent jumps, which are a cause of high risks in stock markets. Therefore, examining the fluctuations in the Chinese stock market to elucidate its gradual changes may promote rational investment behavior, capital operation, and stable market development. Overall, investigating jumps in high-frequency data for the Chinese stock market, which is characterized by unusual price movements, may contribute to both practice and theory.

In contrast to most previous China-based studies that have applied parametric methods, the present study uses the activity signature function (ASF), a nonparametric method based on high-frequency data that was proposed by Todorovand Tauchen (2010) [1], to estimate the activity index of the continuous maincontract price of the China Securities Index (CSI) 300 stock-index futures and assess stochastic process-based stock models (which examine jump diffusion and 
pure jumps) that are applicable to the Chinese stock market. On the basis of two-dimensional realized power variation proposed by Jacod and Todorov (2009) [2], a ratio analysis is subsequently conducted on two independent realized power variations to derive a ratio analysis method for cojumps. This method can be employed to determine the level of cojumps between different stochastic processes.

\section{Literature Review}

\subsection{Jump-Diffusion Models and Pure-Jump Models Based on Stochastic Processes}

Identifying whether stochastic processes involve any jumps is crucial because jumps contain information pertaining to risk management, option pricing, investment portfolio selection, and optimal hedging strategies. In estimation, the quantiles derived from continuous and continuous-jump distributions differ. If changes in the price of an asset involve jumps, then the selection of investment portfolios can be improved considerably. Utilizing information indicated by jumps in volatility and price processes, Liu, and Pan [3] investigate information about investment strategy selection.

Numerous scholars have noted the significance of jumps. In modeling markets, Bollerslev et al. (2008) [4] suggest that jump processes are a crucial source of information for modeling the nondiversible risks of financial markets. In the case of a price jump, policymaking decisions in response to variations in jumps should be made in a timely manner to prevent market chaos. This necessitates analyzing the time-series behavior of jump processes. Introducing jumps into asset pricing models yields considerable value but also incurs certain problems. Cont and Tankov (2003) [5] compare problems associated with jump processes, suggesting that the presence of jumps indicates that a market is incomplete and makes it difficult to process market data. Heston (1993) [6] argues that twosided jump models are affine models and therefore allow for simplified formal solutions to asset prices and their closed-form derivatives. Moreover, two-sided jump models assume that the processes of asset prices and price variations contain rare jumps, such as compound Poisson processes (finite-activity jump processes). Several empirical studies have used two-sided jump models with compound Poisson processes (Eraker et al., 2003) [7]. In an empirical study, Wu (2011) [8] allows for infinite activity in jumps. Thus, many subsequent studies have applied jump models with stochastic volatility. The efficient method of Markov chain Monte Carlo (MCMC) methods (Eraker et al., 2003) [7] have been used to estimate the stochastic volatility jump model for the S \& P 500 index.

Virtually all studies have determined asset prices through jump-diffusion asset price models. However, several studies have shown that asset price stochastic processes can be modeled on the basis of pure-jump processes. Geman (2003) [9] incorporates pure-jump Lévy processes into a stock market pricing model and option prices. Barndorff-Nielsen and Shephard (2001) [10] propose a nonGaussian Ornstein-Uhlenbeck (OU)-based model for estimating stock prices 
and their implied volatility; this model, which contains no diffusion constituent, comprises only one pure-jump Lévy process with nonnegative increments (or subordinate process). The simulation of changes in financial-market asset prices through pure-jump Lévy processes has become a prominent research topic.

\subsection{Stochastic Process Models for Volatility Processes}

The concept of stochastic volatility is instrumental in research into financial market volatility, which provides information on asset selection and pricing. As early as the 1960s, Fama (1965) [11] suggests that the variation of opportunistic prices and the volatility of earnings occur in a stable or volatile manner. Therefore, opportunistic prices exhibit clustering and their variance is time-dependent, indicating that the prices and their variance change stochastically, allowing them to be modeled on the basis of the theory of stochastic processes.

Stochastic process models for volatility processes can be divided into three types: affine jump-diffusion, non-Gaussion OU, and index-type stochastic volatility models. The affine jump-diffusion model contains a non-negative-jump Lévy process with finite variance, $L_{t}$. The model is extensively used to construct equilibrium asset pricing models and simplified asset pricing models (Merton, 1976 [12]; Duffie et al., 2000 [13]). In most applications of the model, $L_{t}$ is a compound Poisson process. Moreover, diffusion and jump constituents coexist in the model; the model is a continuous-jump process.

The non-Gaussion OU model [10] contains a pure-jump Lévy process with nonnegative increments, $L_{t}$, which is also referred to as a subordinate process.

The index-type stochastic volatility model is an EXP-OU- $\Gamma$ model, which includes the process $\Gamma_{t}$. If $\Gamma_{t}$ follows a Brownian motion, then the model is the discrete exponential generalized autoregressive conditional heteroskedasticity (GARCH) model with a continuous-time limit, which is proposed by Nelson (1991) [14].

Whaley (1993) proposes compiling market volatility indices to measure market volatility [15]. In the same year, the Chicago Board Options Exchange (CBOE) launched the VIX index, a volatility index based on the S \& P 100 index (a subset of the S \& P 500 index). The VIX index calculates the implied volatility of eight call and put options for the first- and second-month expirations to estimate the volatility of the S \& P 100 index over the next 30 days. Therefore, this index approximates market volatility processes. Todorov and Tauchen (2010) [1] empirically examine the VIX index, depicting the volatility of the U.S. financial market as a pure-jump process and showing a strong negative correlation between the price and volatility processes of U.S. stocks. No stock warrant market has been established in China, rendering it impossible to estimate the implied volatility of the Chinese stock market. Therefore, the estimation of market volatility is based on its historical or realized volatility. Few studies have modeled market volatility on the basis of stochastic processes.

\subsection{Jump Test}

The selection of asset pricing models is based on the movement of stock prices, 
underlining the need to detect and categorize stock-price jumps. Stock-price jumps can be detected through parametric methods such as the GARCH model or nonparametric means such as the Barndorff-Nielsen and Shephard (BNS) method and threshold multipower variation (TMPV). Parametric methods applied in empirical studies are based on models with unknown parameters that are estimated through regression analysis. If used to estimate intermediateparameters, such methods cause considerable measurement errors, undermining the accuracy of results. Nonparametric methods have comparable advantages.

The BNS method, which is an econometric method for testing jumps, is extensively used for nonparametrically analyzing jump behaviors. Huang and Tauchen (2005) [16] improve this method. Bollerslev et al. (2008) [4] use the method to investigate jumps in the earnings of the U.S. stock market. Ait-Sahalia and Jocad (2009) detect jumps on the basis of TMPV statistics for daily earnings and the asymptotic distribution of the statistics [17] [18].

Cao et al. (2010) [19] extend the Merton model under a jump-diffusion model to propose a jump-diffusion model of power law. Chen and Wang (2010) [20] conduct a systematic analysis of the continuous and jump volatility of the Chinese stock market and use bipower variation with nonparametric methods and high-frequency data on the CSI 300 index to derive the asymptotic statistics of continuous and jump components of market volatility.

\subsection{Cojumps between Price Processes}

Past studies on jumps have also investigated cojumps between different pricing processes. Relevant studies outside of China have focused predominantly on the cojump between constituents in stock market indicies, individual stocks, industry indices, stock and bond markets, and different currency exchange rates. Gilder (2009) [21] identifies large cojumps between individual stocks in the U.S. stock market, suggesting that positive and negative cojumps are symmetrical in number and variation, and that jumps can be dispersed in the market. This finding informs risk management. Dungey and Hvozdyk (2012) [22] use the BNS method to test the cojump between spot and options markets for U.S. bonds, suggesting that cojumps normally occur in short-term options and highfrequency data. Moreover, although the BNS method tests cojumps nonparametrically and uses high-frequency data, it yields jump comovement results on a daily basis, resulting in the loss of real-time correlations between jumps included in high-frequency data. Therefore, the method can be used to test cojumps for financial markets with temporal asynchronicity. Jaeod and Todorov (2009) [2] examine the comovement between individual stocks in the U.S. stock market, proposing the concept of multidimensional realized power variation to estimate the volatility of all subprocesses. Their work provides a basis for analyzing cojumps nonparametrically by using high-frequency data. In summary, the analysis of the comovement between financial markets typically focuses on their cojumps because volatile jumps (rather than continuous changes) explain the relationship between the markets. 
The economic fundamental hypothesis and market contagion hypothesis are two prevailing views on the mechanism underlying the comovement between financial markets. The economic fundamental hypothesis, proposed with the conventional financial theory that investors are rational, applies to the interpretation of the long-term comovement between financial markets. Specifically, under the efficient markets hypothesis, all investors accurately predict future cash flows from assets according to market information, determine a discount rate that accurately reflects the risk overflow, and discount the predicted future cash flows from assets to derive the intrinsic value of the assets. Under this hypothesis, the comovement between the price processes of different assets is associated with the fundamentals of the assets, and the economic foundation of a country depends on the price volatility of its capital market. This explains the inevitable comovement of volatility between domestic financial markets. Thus, the economic fundamental hypothesis accounts for long-term comovements between stock markets.

The market contagion hypothesis, which is based on the framework of behavioral finance, does not account for economic foundation; instead, it examines the investment behavior of individual investors at a microscopic level to interpret investment behavior at the macroscopiclevel of financial markets. Forbes and Rigobon (2002) [23] elucidate this hypothesis, arguing that when a financial market is impacted, the comovement between the price processes of different markets strengthens considerably, and the more sensitive the financial market is, the stronger its comovement with others will be. However, in contrast to conventional financial theories, the hypothesis does not assume that all investors are rational, because cojumps are the result of finite rational decisions made by market participants; this indicates that information asymmetry exists in transactions in financial markets and investors, with limitations on time, effort, and the means for acquiring market information, are likely to engage in identical transactions, exhibiting convergent or herd behavior. In addition, under this hypothesis, nonfundamental factors such as market participants' emotions and market friction have a decisive influence on cojumps.

Essentially, parametric methods are based on models with unknown parameters that are estimated through methods such as regression analysis. If used to estimate intermediate parameters, the methods cause a considerable increase in the number of errors, thus undermining the accuracy of the results. Take for example a typical parametric method, the Granger causality test, which examines the correlation between two processes and regresses it in four steps. The method determines whether two processes correlate with each other, and it identifies the path of influence between both processes. However, it entails multiple regression analysis, in which the intermediate parameters are estimated and therefore the number of errors increases. By contrast, nonparametric methods eliminate the need to estimate any intermediate parameters. They involve only a correlation analysis of the data, thus simplifying the research effort, decreasing the number of errors substantially, and improving the robustness of any findings. 
In summary, in contrast to research conducted outside of China, in which the discussion of comovements between different stochastic processes has increasingly focused on cojumps, studies in China have focused more on parameterization than on jumps or the use of models designed to analyze comovements between two stochastic processes.

\section{Methods}

\subsection{Assumption}

Delbaen and Schachermayer (1994) [24] indicate that if arbitrage-free assumptions are satisfied, then any price processes for transactional securities must be semimartingales. Arbitrage-free assumptions underpin most studies on financial markets; therefore, the present study argues that financial markets in China meet these assumptions and that price processes for the domestic stock market and stock-index futures market are all semimartingales. The most common semimartingale used in financial-market research is the Ito semimartingale, whose characteristic curve, drift, diffusion, and jump compensation are absolutely continuous in time. Models in a semimartingale framework are differentiated by continuous martingales or jumps. Three typical models within the framework are as follows:

1) Continuous model

$$
X_{t}=\int_{0}^{t} b_{1 s} \mathrm{~d} s+\int_{0}^{t} \sigma_{1 s} \mathrm{~d} W_{s}
$$

where $W_{t}$ is a standard Brownian motion.

2) Pure-jump model

$$
\begin{aligned}
Y_{t}= & \int_{0}^{t} b_{2 s} \mathrm{~d} s+\int_{0}^{t} \int_{\mathbb{R}} \sigma_{2 s-} k(y) \tilde{\mu}(\mathrm{d} s, \mathrm{~d} y) \\
& +\int_{0}^{t} \int_{\mathbb{R}} \sigma_{2 s-} k^{\prime}(y) \mu(\mathrm{d} s, \mathrm{~d} y)
\end{aligned}
$$

where $k(y)$ is a continuous truncated function and $k^{\prime}(y)=y-k(y)$.

3) Continuous-jump model

$$
Z_{t}=X_{t}+Y_{t}
$$

\subsection{ASF}

Intuitively, activity describes the volatility of a process or the roughness of a process trajectory. Stochastic processes differ in volatility and activity. The classical Blumenthal-Getoor index, proposed by Blunmenthal and Getoor (1961) [25], describes the activity of pure-jump processes. Ait-Sahalia and Jacod (2007) [26] extend this index, developing the generalized Blumenthal-Getoor index, which can be applied to all time-continuous processes. In statistics, the generalized Blumenthal-Getoor index is formally defined as follows. The scalar process $X$ is observed in a time interval $[0, T]$. At each subinterval $[t-1, t]$ of the interval, $t$ is an integer. With $\Delta_{n}$ as a sampling interval, the high-frequency observed value for process $X$ is derived; specifically, the value of $X$ is observed at the temporal point of $t-1, t-1+\Delta_{n}, \cdots, t-1+\left[1 / \Delta_{n}\right] \Delta_{n}$ in a subinterval, whose dura- 
tion spans 1 day, 1 week, or 1 month. In addition, the activity of process $X$ can be defined at a subinterval $[t-1, t]$ according to the following equation (Ait-Sahalia and Jacod, 2009; Todorov and Tauchen, 2010) [1] [18] [19]:

$$
\beta_{X, t}:=\inf \left\{p>0: p \lim _{\Delta_{n} \rightarrow 0} V_{t}\left(X, p, \Delta_{n}\right)<\infty\right\},
$$

where $V_{t}\left(X, p, \Delta_{n}\right)$ is the power variation of process $X$ at interval $[t-1, t]$, which is expressed by Equation (5):

$$
V_{t}\left(X, p, \Delta_{n}\right)=\sum_{i=1}^{\left[1 / \Delta_{n}\right]}\left|X_{t, i}\right|^{p}, \quad p>0, t \in(0, T]
$$

where $x_{t, i}=X_{t-1+i \Delta_{n}}-X_{t-1+(i-1) \Delta_{n}}$. Power variation explains the overall level of volatility within a specific period. Barndorff-Nielsen and Shephard (2003) [27] have argued for the importance of the power variation Equation (5) in financial economics.

Jacod and Shiryaev (2003) [28] verify that the activity index of semimartingales lies within the interval $[0,2]$. Each semimartingale can be divided into drifts and continuous and discontinuous local martingales. These semimartingale components are ranked according to the level of activity: finite-activity jumps (activity level $=0$ ) such as compound Poisson processes, infinite-activity finite-variation jumps (activity level $=[0,1]$ ), infinite-variation jumps (activity level $=[1,2]$ ), and continuous semimartingales (activity level $=2$ ). Moreover, the activity level of a semimartingale is determined by the activity of its most active component. For example, if process $X$ is driven by a Brownian motion and jump process, then the activity level of the process is 2 (equivalent to that of the continuous martingale).

If process $X$ is not a semimartingale, its activity level is determined in a different manner. For example, if process $X$ is an OU process driven by a fractional Brownian motion, then its activity level is determined by the level of integrity $\left(\delta\right.$ ) of a fraction, namely, $\frac{1}{\delta+0.5}$. Corcuera et al. (2006) [29] demonstrate how the activity level of process $X$ is determined in such case.

Finally, the activity level function is defined at time interval $[t-1, t]$, instead of at all sampling intervals. This allows the activity level of process $X$ to vary with time. Ideally, the activity index should be the same for each time interval.

Todorve and Tauchen [1] discuss a nonparametric method for estimating the activity index; this method, called the ASF, simplifies the estimation of the index. The ASF is expressed as follows. $V_{t}\left(X, p, k \Delta_{n}\right)$ is the power variation defined by Equation (5) at sampling interval $k \Delta_{n} ; \beta_{X, t}$ in Equation (4) is estimated at period $t$, which spans 1 day or 1 month; and if $\Delta_{n} \rightarrow 0$, then a convergence analysis is performed on $V_{t}\left(X, p, k \Delta_{n}\right)$, as expressed by the following equations:

1) Continuous process

$$
\Delta_{n}^{1-p / \beta_{X, T}} V_{T}\left(X, p, \Delta_{n}\right) \stackrel{\text { u.c.p }}{\longrightarrow} A_{p} \int_{0}^{t}\left|\sigma_{1 u}\right|^{p} \mathrm{~d} u
$$

2) Pure-jump process 


$$
\left\{\begin{array}{l}
\Delta_{n}^{1-p / \beta_{Y, T}} V_{T}\left(Y, p, \Delta_{n}\right) \stackrel{\text { u.c.p }}{\longrightarrow} \int_{0}^{t}\left|\sigma_{2 u}\right|^{p} g_{p}\left(a_{s}\right) \mathrm{d} u, \quad p<\beta_{X, T} \\
V_{T}\left(Y, p, \Delta_{n}\right) \stackrel{\text { u.c. }}{\longrightarrow} \sum_{s \leq t}\left|\Delta X_{s}\right|^{p}, \quad p>\beta_{X, T}
\end{array}\right.
$$

3) Continuous-jump process

$$
\left\{\begin{array}{lc}
\Delta_{n}^{1-p / 2} V_{T}\left(Z, p, \Delta_{n}\right) \stackrel{\text { u.c.p }}{\longrightarrow} A_{p} \int_{0}^{t}\left|\sigma_{1 u}\right|^{p} \mathrm{~d} u, & 0<P<2 \\
V_{T}\left(Z, 2, \Delta_{n}\right) \stackrel{\text { u.c.p }}{\longrightarrow} \int_{0}^{t}\left|\sigma_{1 u}\right|^{2} \mathrm{~d} u+\sum_{s \leq t}\left|\Delta Z_{s}\right|^{2}, & P=2 \\
V_{T}\left(Z, p, \Delta_{n}\right) \stackrel{\text { u.c.p }}{\longrightarrow} \sum_{s \leq t}\left|\Delta Z_{s}\right|^{p}, & P>2
\end{array}\right.
$$

with the continuous-jump process, power variation is determined by the continuous martingale when $\mathrm{P}<2$, by continuous martingales and jumps when $\mathrm{P}=2$, and by the jump process when $\mathrm{P}>2$.

The convergence analysis yields Equation (9):

$$
k^{1-\frac{p}{\beta_{\Upsilon, t}}} \Delta_{n}^{1-\frac{p}{\beta_{\Upsilon, t}}} V_{t}\left(\Upsilon, p, k \Delta_{n}\right) \stackrel{P}{\longrightarrow} \Phi_{T}(p), \Delta_{n} \rightarrow 0
$$

where $\Upsilon$ is $X, Y$, or $Z$. When $\Delta_{n} \rightarrow 0$, the above equation is established. The limit of the terms on the right side of Equation (9) is related only to power $p$ and does not depend on other parameters in a stochastic process. Therefore, if $p$ is fixed, then the terms on the left side of the equation converge to the same value, regardless of the value of $k$. Furthermore, let the sampling intervals of two scales be $\Delta_{n}$ and $k \Delta_{n}$; then, the terms on the right side of the equation converge to the same value when $\Delta_{n} \rightarrow 0$, as expressed by Equation (10):

$$
\frac{\left(\Delta_{n}\right)^{1-\frac{p}{\beta_{\Upsilon, t}}} V_{t}\left(\Upsilon, p, \Delta_{n}\right)}{\left(k \Delta_{n}\right)^{1-\frac{p}{\beta_{\Upsilon, t}}} V_{t}\left(\Upsilon, p, k \Delta_{n}\right)} \stackrel{P}{\longrightarrow} 1
$$

After the logarithm of the fraction is taken, the asymptotic value of the equation becomes 0, yielding the implicit function $\beta$, which is solved to obtain Equation (11):

$$
b_{\Upsilon, t}(p)=\frac{\ln (k) p}{\ln (k)+\ln \left[V_{t}\left(\Upsilon, p, k \Delta_{n}\right)\right]-\ln \left[V_{t}\left(\Upsilon, p, \Delta_{n}\right)\right]}, \quad p>0
$$

where $b_{\Upsilon, t}(p)$ is the ASF, and information about the activity level of process $\Upsilon$ is contained in the ASF when $p \in(0,4]$.

The convergence analysis of Equation (11) by Todorov and Tauchen (2010) [1] indicates that when the sampling frequency increases constantly (namely, $\Delta_{n} \rightarrow 0$ at fixed interval $[t-1, t])$, the behavior of $b_{\Upsilon, t}(p)$ is expressed as follows:

1) $b_{\Upsilon, t}(p) \rightarrow 2, \forall p>0$, if $\Upsilon$ contains only continuous martingales;

2) $b_{\Upsilon, t}(p) \stackrel{p}{\longrightarrow} \max (p, 2), \forall p>0$, if $\Upsilon$ contains continuous martingales with jumps; and

3) $b_{\Upsilon, t}(p) \stackrel{p}{\longrightarrow} \max \left(p, \beta_{\Upsilon, t}\right), \forall p \neq \beta_{\Upsilon, t}$, if $\Upsilon$ contains only pure-jump processes.

Where $b_{X, t}(p)$ is locally uniform in the convergence of $p$. The right-hand 
side of the equations above describes the asymptotic behavior of the ASF. For example, to obtain the result in [1], Equation (6) can be used to yield

$$
V_{t}\left(X, p, 2 \Delta_{n}\right) \approx\left(2 \Delta_{n}\right)^{\frac{p}{2}-1} \Phi_{t}(p) \text { and } V_{t}\left(X, p, \Delta_{n}\right) \approx\left(\Delta_{n}\right)^{\frac{p}{2}-1} \Phi_{t}(p) .
$$

Subsequently, simple algebra is used to derive an asymptotic limit of 2 for $b_{X, t}(p)$ in this case. Similar convergence analyses lead to the limits in the other two cases. Moreover, because the ASF is a graphical nonparametierized method (Todorovand Tauchen, 2010) [1], the ASF image of a process indicates which stochastic process the process belongs to.

In a limited sample, the realization of $b_{X, t}(p)$ is the infinitely differentiable function of $p$. An asymptotic analysis suggests that an inflection point may exist in the proximity of $p \approx \beta_{X, t}$, the $p$-image of $b_{X, t}(p)$, and the kurtosis of the point reflects the precision of activity-level estimation. In addition, when $\mathrm{p}>2$, the behavior of the ASF determines whether any jumps exist in process $\mathrm{X}$ at interval $[t-1,1]$ and how strong these jumps are; in addition, it suggests that process $\mathrm{X}$ is established even when it is controlled by a semimartingale, and that jump components are extremely weak (if they meet the Poisson process). Thus, if a function is created with $p$ as an independent variable and $b_{\Upsilon, t}(p)$ as a dependent variable, as indicated by Equation (11), then the ASF can be adopted to estimate the activity index of process $\Upsilon$ and identify the semimartingale of $\Upsilon$.

The ASF images of a stochastic process with only a continuous martingale, a pure-jump stochastic process, and a continuous-jump stochastic process are shown respectively in Figures 1-3.

Outliers are removed from the results to ensure that the ASF is robust. Todorovand Tauchen (2010) [1] introduce the concept of quantiles into the ASF. Specifically, let

$$
B_{q}(p)=\left\{b_{X, t}(p)\right\}_{t=1,2, \cdots, N} \text { The } q \text { th quartiles, } q \in(0,1)
$$

which denote the $q$ th quantile of $b_{X, t}(p)$ for each power $p . B_{q}(p)$ is defined as the quantile ASF (QASF). Over the range of $p \in(0,4]$, the most informative plots are derived from the lower and upper quartiles: $B_{0.25}(p), B_{0.75}(p)$, and the median $B_{0.50}(p)$. Robust methods, such as using quantiles to eliminate

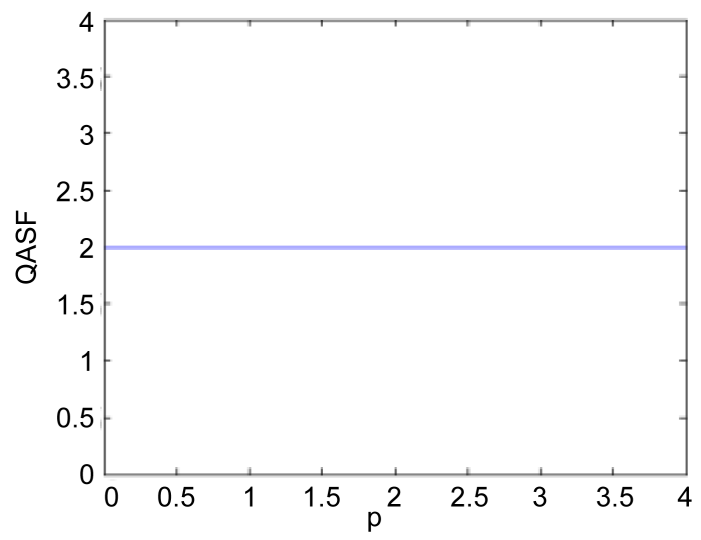

Figure 1. ASF image of a continuous process. 


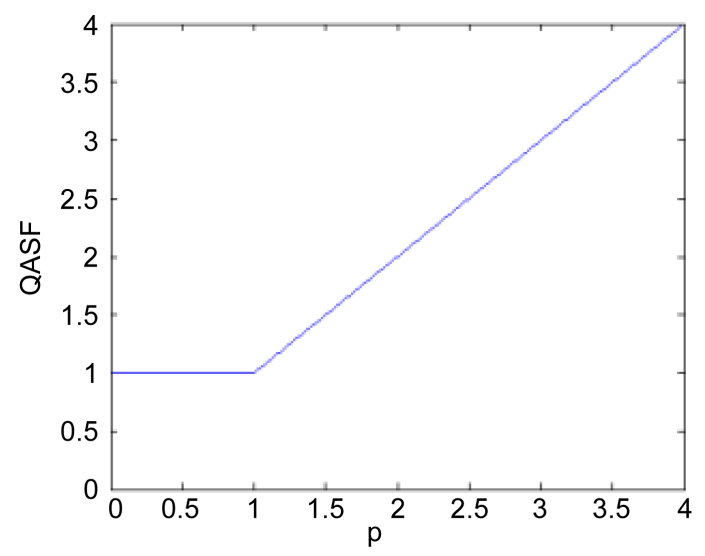

Figure 2. ASF image of a pure-jump process.

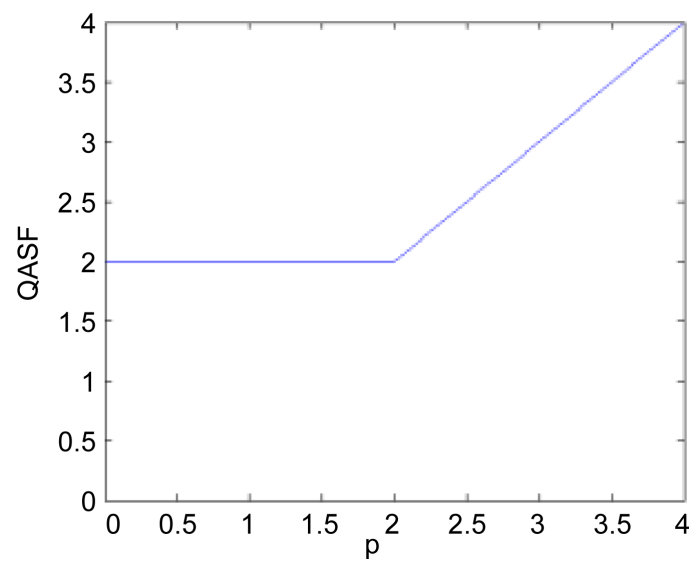

Figure 3. ASF image of a continuous-jump process.

outliers, are crucial to the processing of datasets with extreme observations. In an empirical analysis through the QASF, the activity index of a stochastic process can be estimated by observing the image $B_{0.5}(p)$ and the existence of jumps can be determined by observing the curve slope on the image $B_{0.75}(p)$.

\subsection{Ratio Analysis of Cojumps}

In their analysis of cojumps between individual stocks in the U.S. stock market, Jaeod and Todorov [2] propose multidimensional realized power variation to estimate the volatility of all subprocesses, which is expressed by Equation (13):

$$
V_{t}\left(X, Y, 2, \Delta_{n}\right)=\sum_{i=1}^{1 / \Delta_{n}}\left|X_{t-1+i \Delta_{n}}-X_{t-1+(i-1) \Delta_{n}}\right|^{2}\left|Y_{t-1+i \Delta_{n}}-Y_{t-1+(i-1) \Delta_{n}}\right|^{2}
$$

where $X$ and $Y$ are the subprocesses of a two-dimensional stochastic process, and $X_{t-1+i \Delta_{n}}-X_{t-1+(i-1) \Delta_{n}}$ and $Y_{t-1+i \Delta_{n}}-Y_{t-1+(i-1) \Delta_{n}}$ are the increments of both subprocesses at neighboring sampling intervals.

On the basis of the concept of two-dimensional power variation, this study derives a method for estimating the level of jump synchronicity in subprocesses. Considering the relationship between a two-dimensional stochastic process and the power variation of its subprocesses, assume that 


$$
R_{X Y}=\frac{V_{t}\left(X, Y, 2, \Delta_{n}\right)^{2}}{V_{t}\left(X, 4, \Delta_{n}\right) V_{t}\left(Y, 4, \Delta_{n}\right)}
$$

namely,

$$
R_{X Y}=\frac{\left(\sum_{i=1}^{1 / \Delta_{n}}\left|x_{t, i}\right|^{2}\left|y_{t, i}\right|^{2}\right)^{2}}{\sum_{i=1}^{1 / \Delta_{n}}\left|x_{t, i}\right|^{4} \sum_{i=1}^{1 / \Delta_{n}}\left|y_{t, i}\right|^{4}}
$$

The numerator is expanded as follows and defined as $R 1$ :

$$
\begin{aligned}
& x_{t, 1}^{4} y_{t, 1}^{4}+x_{t, 2}^{4} y_{t, 2}^{4}+\cdots+x_{t, n}^{4} y_{t, n}^{4}+2 x_{t, 1}^{2} y_{t, 1}^{2} x_{t, 2}^{2} y_{t, 2}^{2}+2 x_{t, 1}^{2} y_{t, 1}^{2} x_{t, 3}^{2} y_{t, 3}^{2} \\
& +\cdots+2 x_{t, n-1}^{2} y_{t, n-1}^{2} x_{t, n-1}^{2} y_{t, n-1}^{2}
\end{aligned}
$$

The denominator is expanded as follows and defined as $R 2$ :

$$
\begin{aligned}
& x_{t, 1}^{4} y_{t, 1}^{4}+x_{t, 1}^{4} y_{t, 2}^{4}+\cdots+x_{t, 1}^{4} y_{t, n}^{4}+x_{t, 2}^{4} y_{t, 1}^{4}+x_{t, 2}^{4} y_{t, 2}^{4}+\cdots+x_{t, 2}^{4} y_{t, n}^{4} \\
& +\cdots+x_{t, n}^{4} y_{t, 1}^{4}+x_{t, 1}^{4} y_{t, 2}^{4}+\cdots+x_{t, n}^{4} y_{t, n}^{4}
\end{aligned}
$$

Because of the existence of an inequality

$$
x_{t, i}^{4} y_{t, j}^{4}+x_{t, j}^{4} y_{t, i}^{4} \geq 2 x_{t, i}^{2} y_{t, i}^{2} x_{t, j}^{2} y_{t, j}^{2}
$$

Therefore, $R_{1} \leq R_{2}$ is established, namely, $0 \leq R_{X Y} \leq 1$.

If $\Delta_{n} \rightarrow 0$ is established, then continuities are expressed by Equation (17):

$$
\left\{\begin{array}{l}
x_{t, i}=X_{t-1+i \Delta_{n}}-X_{t-1+(i-1) \Delta_{n}} \rightarrow 0 \\
y_{t, i}=Y_{t-1+i \Delta_{n}}-Y_{t-1+(i-1) \Delta_{n}} \rightarrow 0
\end{array}\right.
$$

and jumps are expressed by Equation (18):

$$
\left\{\begin{array}{l}
x_{t, i}=X_{t-1+i \Delta_{n}}-X_{t-1+(i-1) \Delta_{n}}=\Delta X \\
y_{t, i}=Y_{t-1+i \Delta_{n}}-Y_{t-1+(i-1) \Delta_{n}}=\Delta Y
\end{array}\right.
$$

If jumps exist in both subprocesses, then the jump synchronicity of the subprocesses occurs in two extreme conditions: all jumps are either synchronous or asynchronous. If $R_{X Y}$ is derived under two extreme conditions, then it is distributed between both outliers under normal conditions.

If all jumps in both subprocesses are synchronous, then

$$
\left\{\begin{array}{l}
x_{t, i} y_{t, i}=\Delta X \cdot \Delta Y \neq 0 \\
x_{t, i} y_{t, j} \rightarrow \Delta \cdot 0=0
\end{array}\right.
$$

With the cross term eliminated, the equation becomes

$$
R_{1}=R_{2} \rightarrow x_{t, 1}^{4} y_{t, 1}^{4}+x_{t, 2}^{4} y_{t, 2}^{4}+\cdots+x_{t, n}^{4} y_{t, n}^{4}
$$

Therefore, $R_{X Y} \rightarrow 1$.

If all jumps in the subprocesses are asynchronous, then

$$
x_{t, i} y_{t, i} \rightarrow 0 \cdot \Delta X=0
$$

Because $\Delta_{n} \rightarrow 0$, there must be 


$$
x_{t, i} y_{t, j}=\Delta X \cdot \Delta Y \neq 0
$$

$R_{1} \rightarrow 0, R_{2} \neq 0$ is established, namely, $R_{X Y} \rightarrow 0$

Then $R_{1} \rightarrow 0, R_{2} \neq 0$ is established, namely, $R_{X Y} \rightarrow 0$.

The analysis of the cojump of both subprocesses involves estimating the synchronicity of jumps and determining the directions of the jumps (namely, whether the jumps correlate positively or negatively). Examining the correlations between jumps is relatively simple when using the proposed method. If $\Delta_{n} \rightarrow 0$ is established, then the scale of the two-dimensional power variation represents the extent to which jumps contribute to the volatility of processes. On the basis of their sign, $x_{t, i}$ and $y_{t, i}$ are split into power variations due to jumps in the same direction and those in the opposite direction, which are denoted respectively as is $V_{t}\left(X, Y, 2, \Delta_{n}\right)^{+}$and $V_{t}\left(X, Y, 2, \Delta_{n}\right)^{-}$. Thus,

$$
V_{t}\left(X, Y, 2, \Delta_{n}\right)=V_{t}\left(X, Y, 2, \Delta_{n}\right)^{+}+V_{t}\left(X, Y, 2, \Delta_{n}\right)^{-}
$$

The ratio of the power variation of jumps in the same direction to that of all jumps indicates that 1) the closer the correlation between jumps in both subprocesses is to 1 , the more positive the correlation is; 2 ) the closer the correlation is to 0 , the more negative it is, and 3 ) a correlation approximating 0.5 suggests no notable positivity or negativity.

$$
r_{X Y}=\frac{V_{t}\left(X, Y, 2, \Delta_{n}\right)^{+}}{V_{t}\left(X, Y, 2, \Delta_{n}\right)^{-}}
$$

\subsection{Instantaneous Historical Volatility}

Volatility is a measure of the fluctuation of target asset-price processes. Actual asset-price processes are stochastic and cannot be estimated accurately. Therefore, actual volatility processes are also stochastic, and each moment of volatility defies accurate estimation. Volatility is calculated in terms of historical, implied, and realized volatility. The estimation of realized volatility, which is based on high-frequency data, is the most suitable for the present study. However, because high-frequency data are essential to volatility analysis and estimating the volatility of these data necessitates data of higher frequency, this study does not simulate actual volatility processes on the basis of realized volatility. Implied volatility, which is derived by incorporating warrant prices into warrant-price models, is the expected value of the actual volatility and has high representativeness. For example, the CBOE developed the VIX index by measuring the volatility of the option prices of the S \& P 500 index, and the VIX index shares numerous statistical characteristics with actual volatility. However, the volatility processes of the Chinese stock market cannot be estimated because no warrant market has been established in China, rendering it impossible to determine the prices of Chinese warrants. Accordingly, the present study estimates the instantaneous historical volatility to simulate actual volatility processes. The estimation is expressed by Equation (25): 


$$
\begin{aligned}
& \sigma_{n}^{2}=\frac{1}{m-1} \sum_{i=1}^{m}\left(u_{n-i}-\bar{u}\right)^{2} \\
& \bar{u}=\frac{1}{m} \sum_{i=1}^{m} u_{n-i}
\end{aligned}
$$

where $\sigma_{n}$ is the volatility between the $n$-1and $n$ sampling points, $S_{i}$ is the asset price at time $t$, and $u_{i}=\ln \left(S_{i} / S_{i-1}\right)$ is the earnings rate. To obtain high-frequency data, the instantaneous historical volatility is estimated using the historical volatility estimates at three sampling intervals as the volatility estimate of 5-minute closing price data at the last time point of the data. In other words, the historical volatility is estimated for each set of three intervals (through the sliding window method), and the estimation results are used as the high-frequency data needed to examine actual volatility processes.

\section{Empirical Results}

This study uses high-frequency data on the CSI 300 index for the period from April 8, 2005 (when the index was published) to December 31, 2012; and on the continuous main-contract prices of the CSI 300 stock-index futures for the period between April 16, 2010 (when the stock-index futures opened) and June 31, 2012. The sampling interval is 5 minutes. ${ }^{1}$ On the basis of the sliding-window estimation of the historical volatility, the historical volatility estimates at three sampling intervals are used as the volatility estimate of 5-minute closing price data at the last time point of the data to replace high-frequency data for examining actual volatility processes. All data are derived from the Wind Economic Database.

\subsection{CSI 300 Index Model}

As illustrated in the previous section, the ASF involves a convergence analysis conducted at the sampling interval $\Delta_{n} \rightarrow 0$. Therefore, when the function is used to test models, the sampling interval must be adequately small relative to the time interval $[t-1, t]$. The 5 -minute sampling interval in this study can be adjusted by setting the time interval to 1 day or 1 month. Accordingly, this study compares the ASF results obtained at daily and monthly intervals to elucidate any limitations regarding the application of the function.

High-frequency discrete data on CSI 300 index processes between April 8, 2005, and December 31, 2012, are analyzed on the basis of the aforementioned procedure to yield three quantiles lines of $b_{X, t}(p)$ at both daily and monthly intervals (Figure 4 and Figure 5, respectively), the results of which suggest that the CSI 300 index process is a continuous-jump process, validating the jumpdiffusion model proposed by Merton (1976) [12], and that pure-jump models are not representative of active stock markets. Moreover, results from the extension of the Merton model are acceptable. Cao et al. (2011) [20] observe that jump sizes and intervals in jump-diffusion models conform to power laws, and they adapt the Merton model to be a power-law jump-diffusion model.

${ }^{1}$ Sampling is conducted at a 5-minute interval to prevent noise due to microstructural noise (Andersen, 2001; Koopman, 2005). 


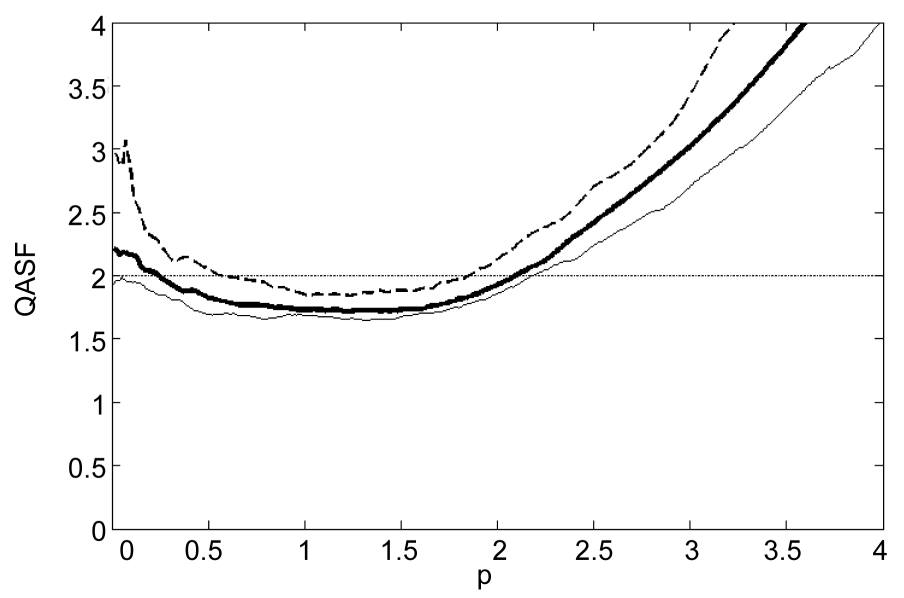

Figure 4. ASF curve for the CSI 300 index at daily intervals (from top to bottom: 0.75 quantile, 0.50 quantile, and 0.25 quantile).

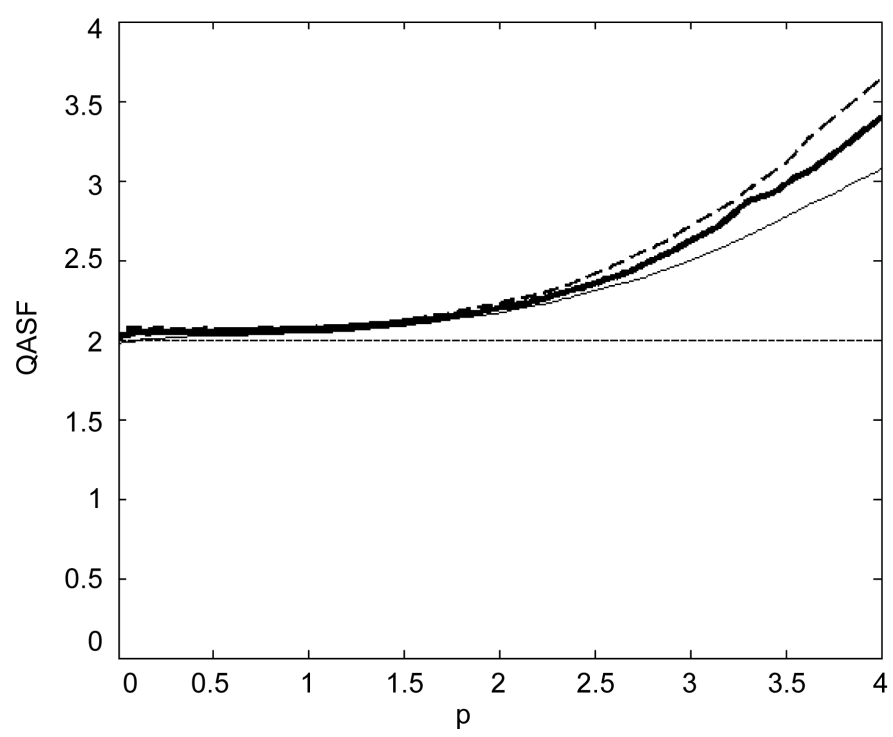

Figure 5. ASF curve for the CSI 300 index at monthly intervals (from top to bottom: 0.75 quantile, 0.50 quantile, and 0.25 quantile).

The high-frequency discrete data are divided into 93 monthly subintervals, and data for each subinterval are processed on the basis of the aforementioned procedure to derive $0.25 \mathrm{ASF}$ quantile curves with daily intervals. The curves are divided into four types, depending on the curvature.

The first curve type depicts the CSI 300 index process as a continuous-jump process (Figure 6(a)). The ASF curves associated with 84 of the 93 monthly subintervals belong to this type, corresponding to the test results of the CSI 300 index model for most of the data. The second curve type characterizes the CSI 300 index process as a pure jump process (Figure 6(b)); this type of curve covers 7 months (July-October 2008, March-April 2009, and January 2010). The pure jump process indicates high volatility or a substantial sudden change in price within stock markets, and this typically occurs in times of market instability, particularly in the 2008 financial crisis. In the third curve type, the CSI 300 index 


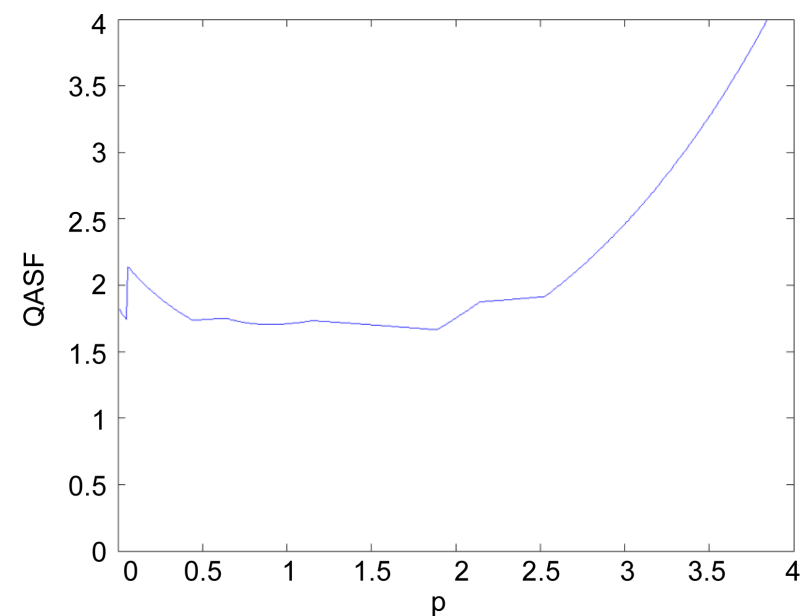

(a)

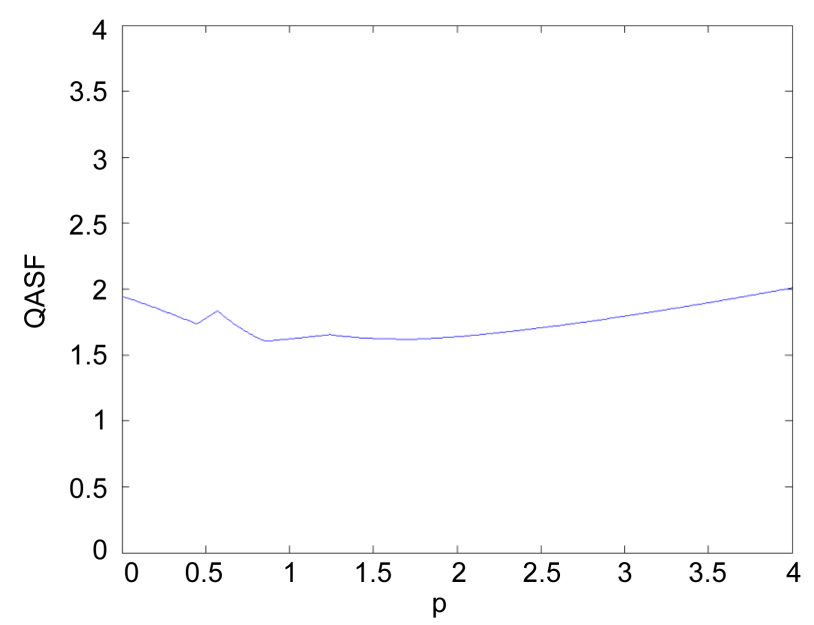

(c)

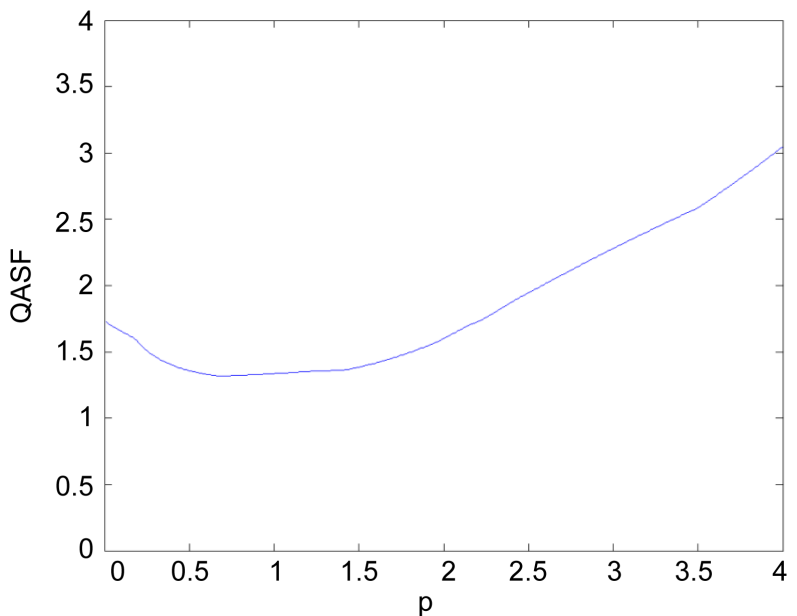

(b)

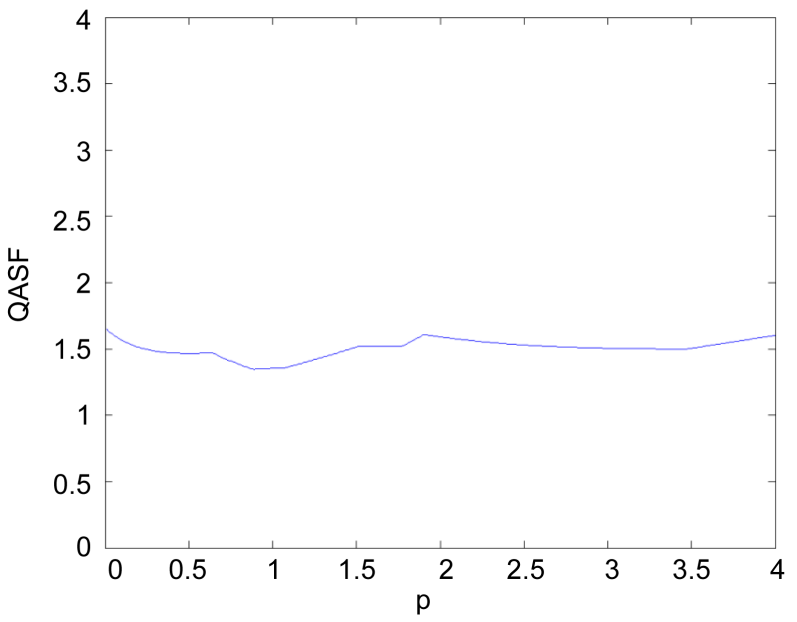

(d)

Figure 6. ASF curves for the CSI 300 index. (a) April 2005; (b) July 2008; (c) September 2005; (d) November 2006.

contains no jump (Figure 6(c)), and the index process remains a continuousmartingale process throughout the 93 monthly subintervals. As such, the stock market shows stable price volatility. This process normally occurs in times of market stability such as in September 2005. In the fourth curve type, the CSI 300 index process is a fractional Brownian motion or a type of non-semi-martingale process (Figure 6(d)). Such a process is evident in data for November 2006; however, it cannot be specifically classified in the present study because the ASF categorizes only semimartingales.

The long-term trend of the CSI 300 index indicates that the price process of the Chinese stock market is a continuous-martingale process with jumps. The short-term trend in the index contradicts the overall trend for certain periods but conforms to most of the other periods, exhibiting normal fluctuations in stock-market prices.

\subsection{Futures Main-Contract Price of the CSI 300 Index}

High-frequency discrete data on the continuous main-contract price process of 
the CSI 300 stock-index futures are analyzed through the procedure described in Section 4.1 to yield three quantiles of $b_{X, t}(p)$ at both daily and monthly intervals (Figure 7 and Figure 8, respectively), suggesting that this continuous maincontract price process is a continuous-jump process. Thus, continuous stochastic processes with jumps describe the volatility of stock-index futures markets, although related studies in the literature are scant.

The high-frequency discrete data spanning from April 16, 2010, to June 31, 2012, are divided into 27 monthly subintervals, and each subinterval is processed

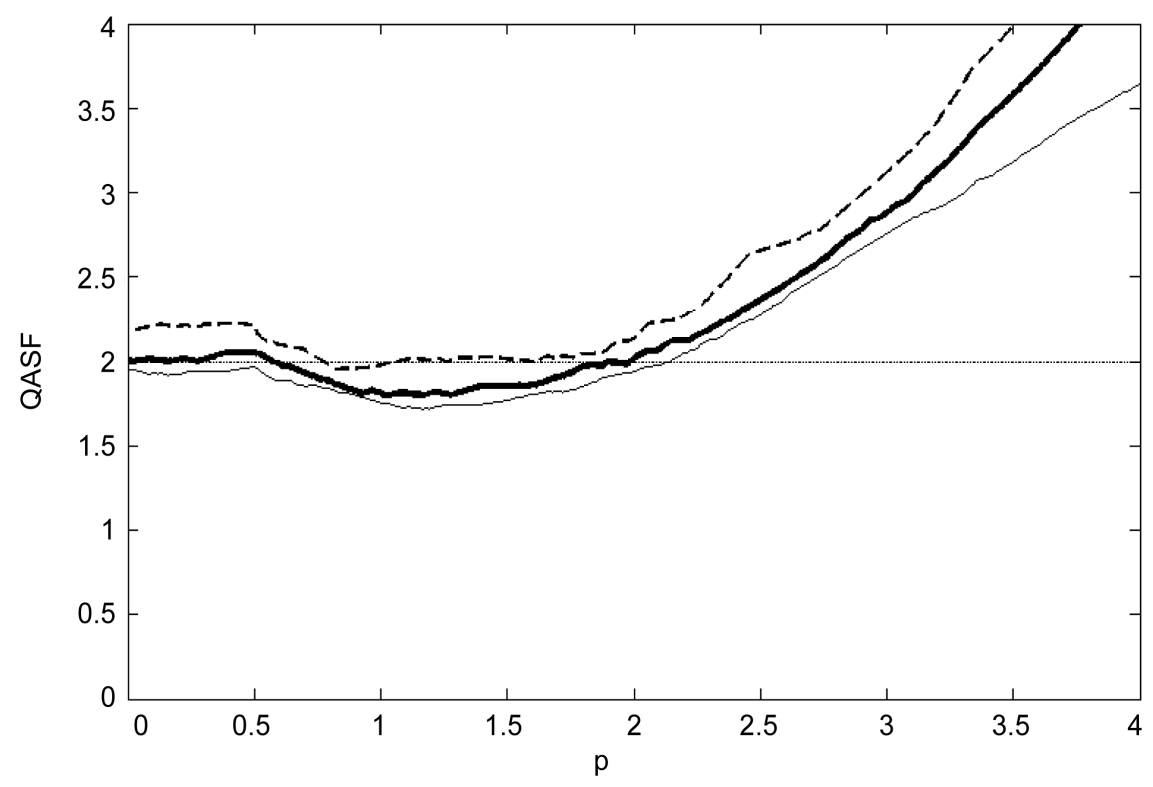

Figure 7. ASF curve for the main-contract price for the CSI 300 stock-index futures at daily intervals (from top to bottom: 0.75 quantile, 0.50 quantile, and 0.25 quantile).

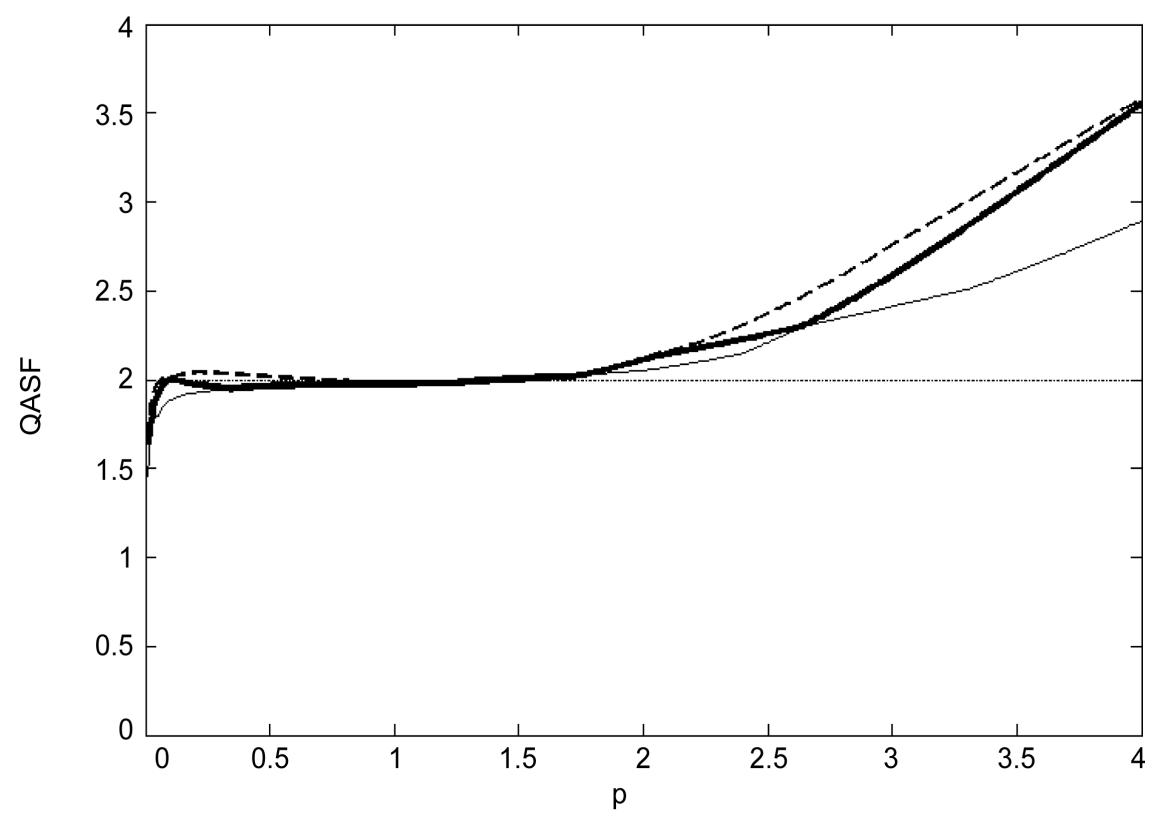

Figure 8. ASF curve for the main-contract price for the CSI 300 stock-index futures at monthly intervals (from top to bottom: 0.75 quantile, 0.50 quantile, and 0.25 quantile). 
through the procedure described in Section 4.1 to derive 0.50 ASF quantile curves with daily intervals. The curves are divided into two types, depending on the curvature. The first curve type describes the continuous main-contract price process of the CSI 300 stock-index futures as a continuous-jump process (Figure 9(a)), which corresponds to the test results of the CSI 300 index model for most of the data. The ASF curves of 23 of the 27 monthly subintervals belong to this type. The second curve type characterizes the continuous main-contract price process of the CSI 300 stock-index futures as a pure jump process, which appears in the ASF curves of the remaining four monthly subintervals. Accordingly, high volatility in the stock-index futures market is concentrated in the first few months of the launch of the market and exhibits some periodic changes within 6 months and 1 year of its launch.

The long-term trend in the continuous main-contract price process of the CSI 300 stock-index futures suggests that the prices of Chinese stock-index futures entail continuous-martingale processes with jumps. The short-term trend in the price process contradicts the overall trend at certain periods but conforms to most of the other periods. Moreover, compared with Figure 3 and Figure 7, Figure 4 and Figure 8 display more notable inflection points on the curves and less space between the quantiles, suggesting that images obtained at monthly intervals $[t-1, t]$ are more accurate than those obtained at daily intervals. The relative sampling interval for the monthly intervals $(1 / 1,056)$ is higher than that for the daily intervals (1/48). Accordingly, the ASF is based on high-frequency data; the higher the frequency is, the more accurate the results derived from the function will be.

\subsection{Volatility of the CSI 300 Index}

The historical volatility of the CSI 300 index is estimated through the ASF to yield three quantiles of $b_{X, t}(p)$ at monthly intervals (Figure 10), suggesting that

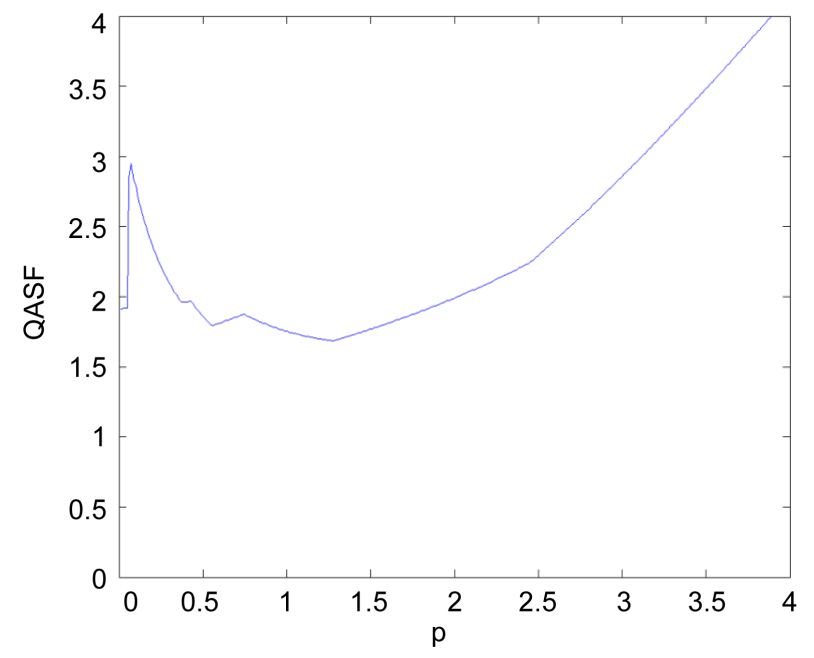

(a)



(b)

Figure 9. ASF curves for the continuous main-contract price process of the CSI 300 stock-index futures; (a) November 2010; (b) September 2010. 


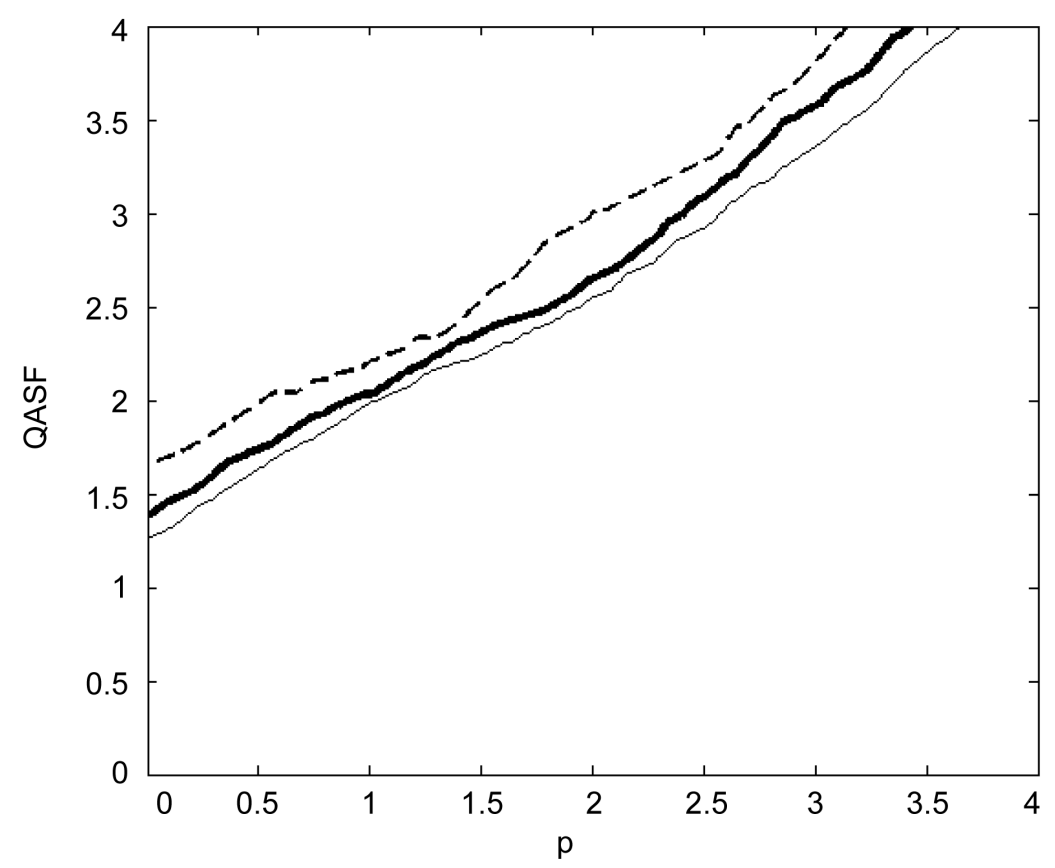

Figure 10. ASF curve for the instantaneous historical volatility of the CSI 300 index.

pure jump processes characterize the volatility of stock market prices. The VIX index, which reflects the volatility of U.S. financial markets, has been proved to be a pure jump process, and the present study shows that the volatility of Chinese financial markets is also a pure jump process. In summary, despite limited data, this study uses not only historical data but also stochastic process models to estimate the volatility of Chinese financial markets.

\subsection{Cojumps}

Cojumps are tested typically through a ratio analysis, which examines the distribution of cojumps expressed as a ratio between 0 and 1 or determines the distance from the mean and median of the ratio at all intervals $t$ to 1 .

The synchronicity of jump processes between CSI 300 future and spot indices is investigated. Under synchronous temporal conditions, $R_{X Y}$ is estimated for each trading day, and its distribution, which ranges between 0 and 1 , is presented in the form of a histogram (Figure 11(a)). The mean of the $535 R_{X Y}$ values is $0.81 ; 489$ of them range between 0.6 and 1 , suggesting that under synchronous temporal conditions, the occurrence of jumps is highly synchronous between the CSI 300 index process and the continuous main-contract price process of the CSI 300 stock-index futures.

Under an asynchronous temporal condition (when the time of stock-index futures surpasses the CSI 300 index by 5 minutes), $R_{X Y}$ is estimated for each trading day, and its distribution, which ranges between 0 and 1 , is presented in the form of a histogram (Figure 11(b)). The mean of the $535 R_{X Y}$ values is 0.665 ; 359 of them range between 0.6 and 1, suggesting that under asynchronous temporal conditions, the occurrence of jumps is highly synchronous between in the 


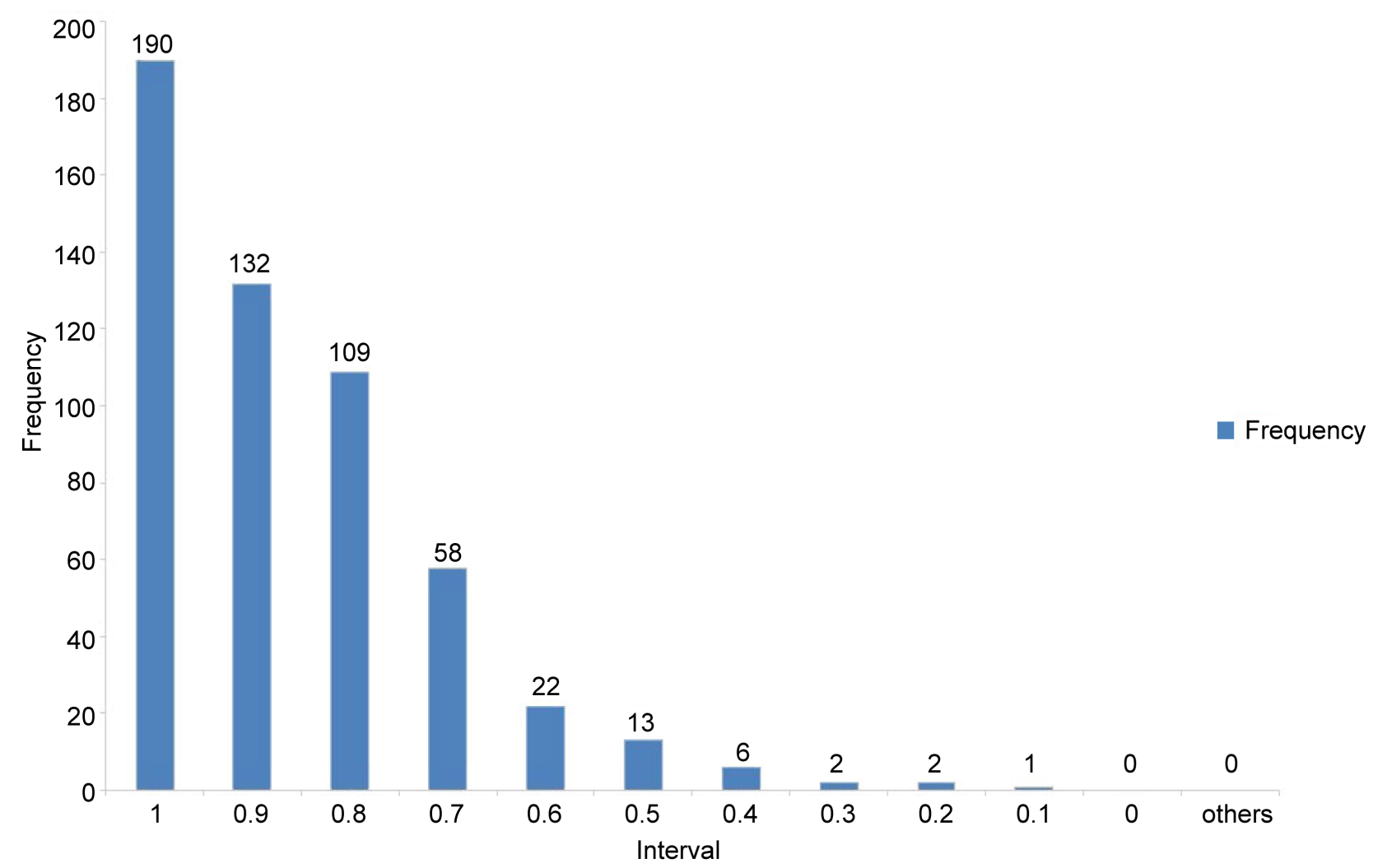

(a)

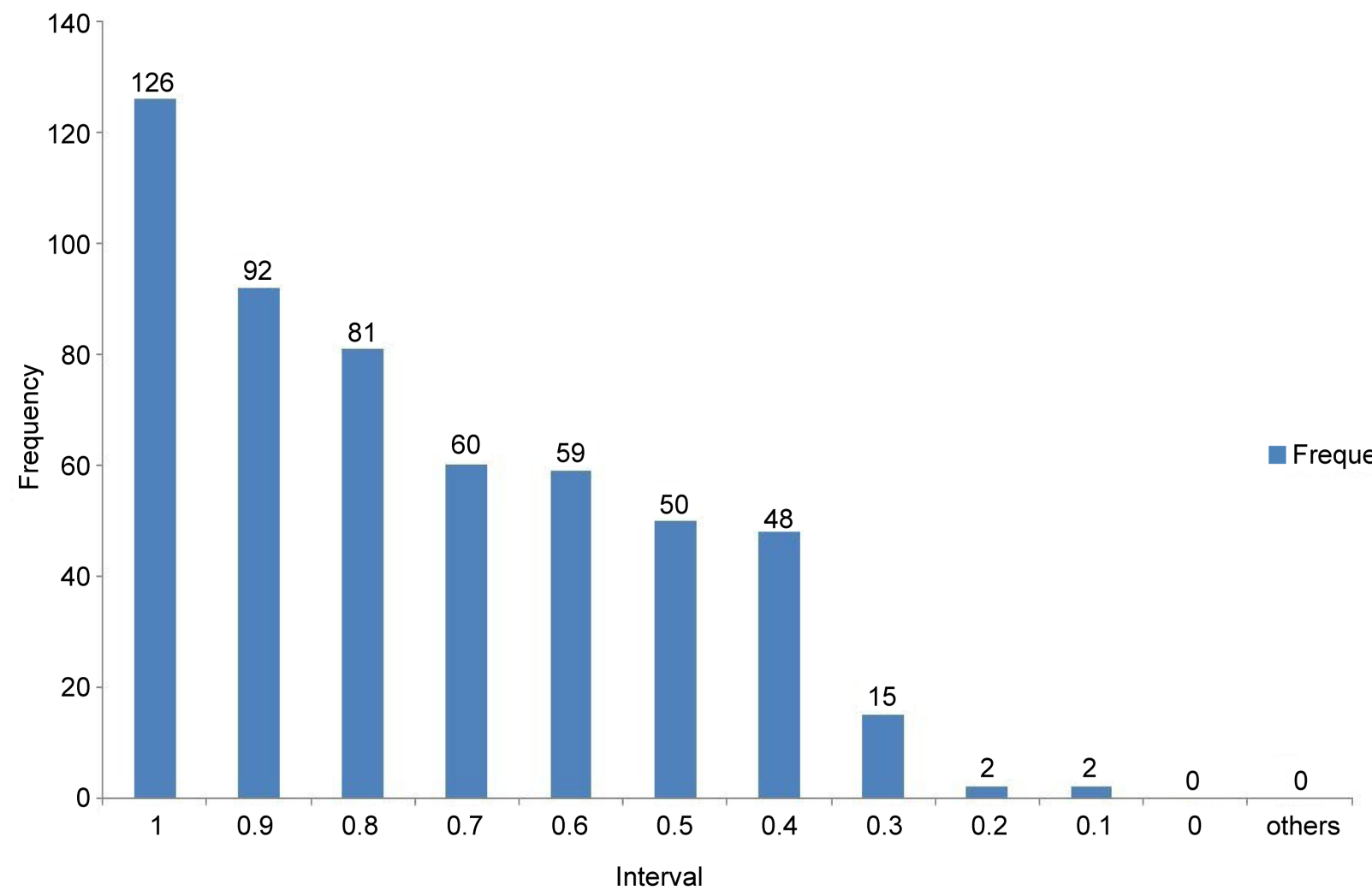

(b)

Figure 11. Histogram of the $R_{X Y}$ distributions of CSI 300 future and spot indices. (a) Synchronous temporal conditions; (b) Asynchronous temporal conditions. 
CSI 300 index process and the continuous main-contract price process of the CSI 300 stock-index futures, but the synchronicity is lower than under synchronous temporal conditions. This finding suggests that stock-index futures prices predict prices in stock markets. Moreover, information technologies are increasingly used in financial markettrading. The ongoing development of Internet technologies has expedited the dissemination of price information among financial markets, allowing investors to synergize information from different markets and make investment decisions in a matter of 5 minutes; this has also shortened the lag time in their response to the markets.

The positive and negative correlations of jumps are tested by estimating $r_{X Y}$ to determine the proportion of positive cojumps among all jumps for a twodimensional process formed by the CSI 300 index process and the continuous main-contract price process of the CSI 300 stock-index futures. The mean and median of $r_{X Y}$ are respectively 0.993 and 0.998 , indicating that the volatility of both processes essentially moves in the same direction and that jumps in the processes show strong positive correlations.

The synchronicity of jumps between the CSI 300 index and its volatility is determined by calculating $R_{X Y}$ for each trading day, the results of which are shown in Figure 12. The mean of the $1,883 R_{X Y}$ values is $0.474 ; 496$ of them range between 0.6 and 1, suggesting low synchronicity in the occurrence of jumps between the CST 300 index process and its volatility process. This undermines the need to examine the positive and negative correlations of jumps for both processes.

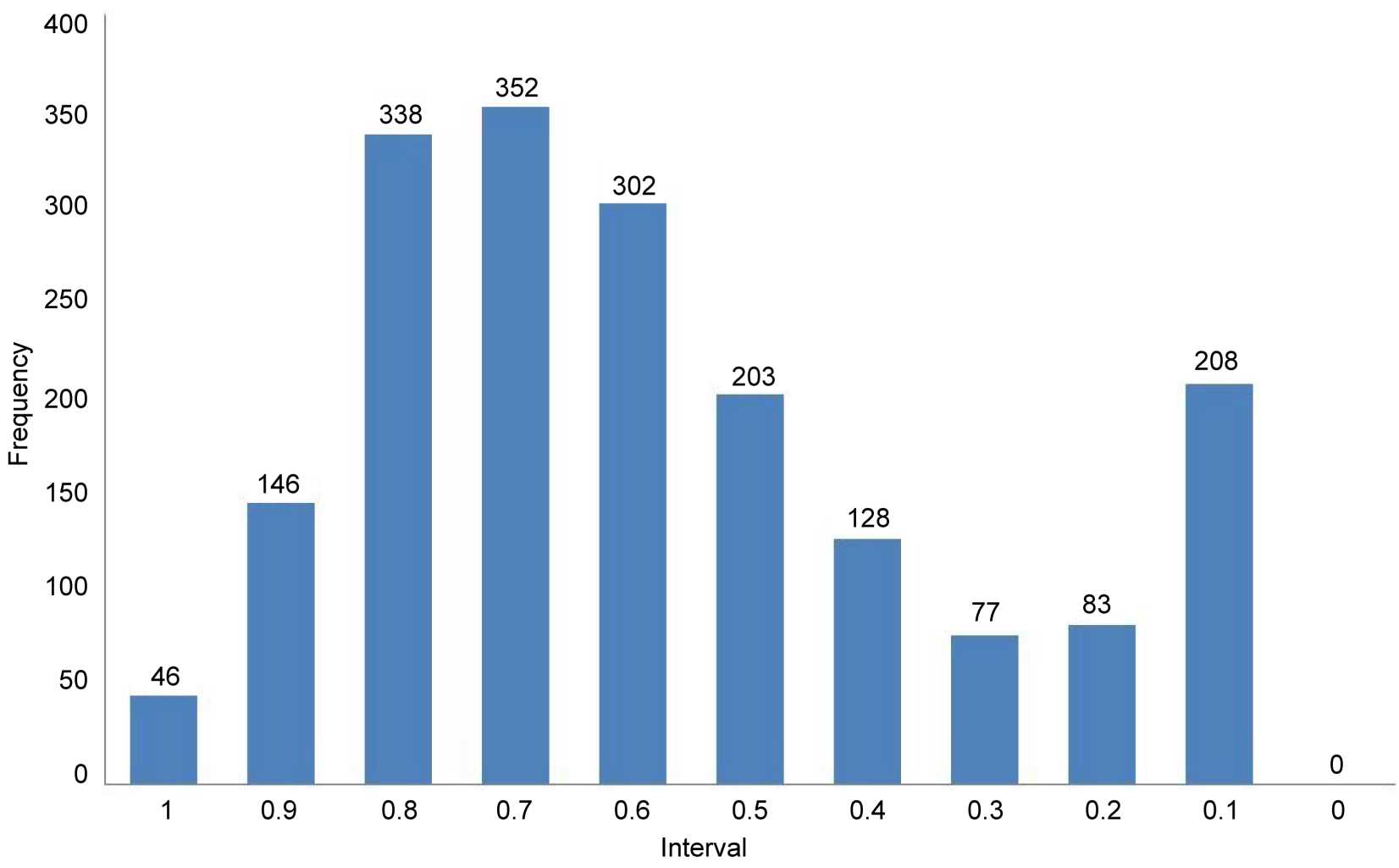

Figure 12. Histogram of the $R_{X Y}$ distributions of the CSI 300 index and its instantaneous historical volatility. 


\section{Conclusions}

The findings of this study yield the following conclusions. First, the CSI 300 index fundamentally involves a continuous-jump process, supporting the jumpdiffusion model proposed by Mertaon (1976) [12]. Moreover, the activity indices of pure-jump models are inconsistent with any actual price processes and are therefore not representative of any active stock markets. In the price process of the Chinese stock market, the form of jump depends on continuous volatility processes; therefore, the analysis and expansion of pure-jump models are based on the Merton model. The CSI 300 index exhibits the characteristics of purejump or continuous processes in certain periods, making it necessary to adjust the price-volatility model for the index on the basis of macroeconomic factors and relevant policies.

Second, the continuous main-contract price process of the CSI 300 stock-index futures agrees with continuous-martingale processes with jumps, indicating that continuous stochastic processes with jumps describe the volatility of stockindex futures markets. This may provide fresh insights into research on stockindex futures markets. Furthermore, in line with the CSI 300 index process, the continuous main-contract price process of the CSI 300 stock-index futures exhibits the characteristics of continuous-jump processes at most times and the characteristics of pure-jump processes at certain times. Thus, the price process model for the CSI 300 stock-index futures should be adjusted depending on actual circumstances.

Third, the volatility process of the CSI 300 index suggests that this process fits pure-jump processes more closely than it does other stochastic processes. This finding corresponds with those of Todorov and Tauchen that the VIX index is a pure-jump process. This contributes theoretically to the predication of the volatility of Chinese financial markets because parameterizing the time of volatility and fitting it with a pure-jump process may reflect the actual market volatility more closely.

Third, the analysis of cojumps suggests that jumps in the CSI 300 index price process and the continuous main-contract price process of the CSI 300 stockindex futures occur simultaneously in most cases (with a time lag of less than 5 minutes) and share a strong positive relationship. As such, the formulation of strategies on risk management and portfolio construction in the stock market or stock-index futures market in China should involve modeling the volatility of different markets. The price process of the Chinese stock market and the price volatility of the market show no notable cojump, although whether such a cojump may ever occur during the development of the market necessitates further research.

\section{Acknowledgements}

This work was supported, in part, by the National Natural Science Foundation of China (Grant Nos. 70801066, 71071167, 71071168, 71371200), and by a grant 
from Sun Yat-sen University Basic Research Funding (Grant Nos. 1009028, 1109115, 16wkjc13).

\section{References}

[1] Todorov, V. and Tauchen, G. (2010) Activity Signature Functions for High-Frequency Data Analysis. Journal of Econometrics, 154, 125-138.

[2] Jacod, J. and Todorov, V. (2009) Testing for Common Arrivals of Jumps for Discretely Observed Multi-Dimensional Processes. Annals of Statistics, 37, 1792-1838. https://doi.org/10.1214/08-AOS624

[3] Liu, J. and Pan, J. (2003) Dynamic Derivative Strategies. Journal of Financial Economics, 69, 401-430.

[4] Bollerslev, T., Law, T. and Tauchen, G. (2008) Risk, Jumps, and Diversification. Journal of Econometrics, 144, 234-256.

[5] Cont, R. and Tankov, P. (2003) Financial Modelling with Jump Processes. Chapman and Hall, Boca Raton, Florida. https://doi.org/10.1201/9780203485217

[6] Heston, S. (1993) A Closed-Form Solution for Options with Stochastic Volatility with Applications to Bond and Currency Options. Review of Financial Studies, 6, 327-343. https://doi.org/10.1093/rfs/6.2.327

[7] Eraker, B., Johannes, M. and Polson, N. (2003) The Impact of Jumps in Volatility and Returns. Journal of Finance, 58, 1269-1300. https://doi.org/10.1111/1540-6261.00566

[8] Wu, L. (2011) Variance Dynamics: Joint Evidence from Options and High-Frequency Returns. Journal of Econometrics, 160, 280-287.

[9] Geman, H. (2003) Pure Jump Levy Processes for Asset Price Modeling. EFA 2003 Annual Conference Paper No. 590.

[10] Barndorff-Nielsen, O. and Shephard, N. (2001) Non-Gaussian Ornstein-UhlenbeckBased Models and Some of Their Uses in Financial Economics. Journal of the Royal Statistical Society Series B, 63, 167-241. https://doi.org/10.1111/1467-9868.00282

[11] Fama, E.F. (1965) The Behavior of Stock-Market Prices. Journal of Business, 38, 34-105. https://doi.org/10.1086/294743

[12] Merton, R. (1976) Option Pricing When the Underlying Stock Returns Are Discontinuous. Journal of Financial Economics, 3, 125-144.

[13] Duffie, D., Pan, J. and Singleton, K. (2000) Transform Analysis and Asset Pricing for Affine Jump-Diffusions. Econometrica, 68, 1343-1376. https://doi.org/10.1111/1468-0262.00164

[14] Nelson, D.B. (1991) Conditional Heteroskedasticity in Asset Returns: A New Approach. Econometrica, 59, 347-370. https://doi.org/10.2307/2938260

[15] Whaley, R.E. (1993) Derivatives on Market Volatility: Hedging Tools Long Overdue. Journal of Derivatives, 1, 71-84. https://doi.org/10.3905/jod.1993.407868

[16] Huang, X. and Tauchen, G. (2005) The Relative Contribution of Jumps to Total Price Variance. Journal of Financial Econometrics, 3, 456-499. https://doi.org/10.1093/jifinec/nbi025

[17] Ait-Sahalia, Y. and Jacod, J. (2009) Estimating the Degree of Activity of Jumps in High Frequency Data. Annals of Statistics, 37, 2202-2244.

https://doi.org/10.1214/08-AOS640

[18] Ait-Sahalia, Y. and Jacod, J. (2009) Testing for Jumps in a Discretely Observed Process. Annals of Statistics, 37, 184-222. https://doi.org/10.1214/07-AOS568 
[19] Cao, H.D., Li, Y. and He, Z. (2011) Stock Pricing Model: Jump Diffusion Model of Power Law. Journal of Management Sciences in China, 14, 46-59.

[20] Chen, G.J. and Wang, Z.H. (2010) Continuous Volatility and Jump Volatility in China's Stock Market. System Engineering Theory and Practice, 30, 1554-1562.

[21] Gilder, D. (2009) An Empirical Investigation of Intraday Jumps and Cojumps in US Equities. Research Paper.

http://citeseerx.ist.psu.edu/viewdoc/download?doi=10.1.1.503.9736\&rep=rep1\&type $=\mathrm{pdf}$

[22] Dungey, M. and Hvozdyk, L. (2012) Cojumping: Evidence from the US Treasury Bond and Futures Markets. Journal of Banking \& Finance, 36, 1563-1575.

[23] Forbes, K.J. and Rigobon, R. (2002) No Contagion, Only Interdependence: Measuring Stock Market Comovements. Journal of Finance, 57, 2223-2261. https://doi.org/10.1111/0022-1082.00494

[24] Delbaen, F. and Schachermayer, W. (1994) A General Version of the Fundamental Theorem of Asset Pricing. Mathematische Annalen, 300, 463-520. https://doi.org/10.1007/bf01450498

[25] Blumenthal, R. and Getoor, R. (1961) Sample Functions of Stochastic Processes with Independent Increments. Journal of Mathematics and Mechanics, 10, 493-516.

[26] Ait-Sahalia, Y. and Jacod, J. (2007) Volatility Estimators for Discretely Sampled Levy Processes. Annals of Statistics, 35, 355-392. https://doi.org/10.1214/009053606000001190

[27] Barndorff-Nielsen, O. and Shephard, N. (2003) Realized Power Variation and Stochastic Volatility Models. Bernoulli, 9, 243-265. https://doi.org/10.3150/bj/1068128977

[28] Jacod, J. and Shiryaev, A.N. (2003) Limit Theorems for Stochastic Processes. 2nd Edition, Springer Verlag, Berlin. https://doi.org/10.1007/978-3-662-05265-5

[29] Corcuera, J., Nualart, D. and Woerner, J. (2006) Power Variation of Some Integral Fractional Processes. Bernoulli, 12, 713-735. https://doi.org/10.3150/bj/1155735933

Submit or recommend next manuscript to SCIRP and we will provide best service for you:

Accepting pre-submission inquiries through Email, Facebook, LinkedIn, Twitter, etc. A wide selection of journals (inclusive of 9 subjects, more than 200 journals)

Providing 24-hour high-quality service

User-friendly online submission system

Fair and swift peer-review system

Efficient typesetting and proofreading procedure

Display of the result of downloads and visits, as well as the number of cited articles

Maximum dissemination of your research work

Submit your manuscript at: http://papersubmission.scirp.org/

Or contact jmf@scirp.org 